\title{
DIFFERENTIAL OPERATORS AND ACTIONS OF LIE ALGEBROIDS
}

\author{
Y. Kosmann-Schwarzbach and K. C. H. Mackenzie
}

\begin{abstract}
We demonstrate that the notions of derivative representation of a Lie algebra on a vector bundle, of semi-linear representation of a Lie group on a vector bundle, and related concepts, may be understood in terms of representations of Lie algebroids and Lie groupoids, and we indicate how these notions extend to derivative representations of Lie algebroids and semi-linear representations of Lie groupoids in general.
\end{abstract}

\section{Introduction}

This paper deals with actions on vector bundles. The first part (Sections 1 13 ) deals with the infinitesimal actions of Lie algebras and Lie algebroids, while the second part (Sections 4 and 5 ) deals with the global actions of Lie groups and Lie groupoids.

When passing from the case of actions on vector spaces to that of actions on vector bundles, the notions of linear endomorphism and linear isomorphism admit straightforward generalizations which, however, have rarely been spelled out in the literature. On the global level, it is clear that the analogue of a linear isomorphism is a vector bundle automorphism, not necessarily base-preserving. Such an automorphism gives rise to an isomorphism of the vector space of sections which has the additional property of semi-linearity, i.e., when a section is multiplied by a function on the base, the image of this section is multiplied by the given function composed with the inverse of the diffeomorphism of the base defined by the vector bundle automorphism. (This could be viewed as a morphism from the space of sections to itself, equipped with two module structures.) Actions of Lie groups on a vector bundle therefore give rise to representations in its space of sections by semi-linear isomorphisms.

The infinitesimal counterpart of such a vector bundle automorphism can be determined by differentiating a one-parameter group of semi-linear isomorphisms of the space of sections. The result is a derivative endomorphism, a first-order differential operator with the additional property that its symbol is a scalar multiple of the identity. Thus the derivative endomorphisms of the space of sections of a

2000 Mathematics Subject Classification. Primary 17B66. Secondary 22A22, 58D19, 58H05. 
vector bundle generalize the linear endomorphisms of a vector space, and the representations of Lie algebras on the space of sections of a vector bundle by derivative endomorphisms generalize the linear representations.

These basic facts (and their cohomological interpretation, which we do not repeat here) were described by Kosmann-Schwarzbach in 1976 [22]. Actually, semilinear isomorphisms and derivative endomorphisms are both particular cases of what Jacobson, in 1935, called pseudo-linear transformations [19], 20]. But Jacobson's purpose was to extend the theory of elementary divisors, and the relevance of his work for differential geometry was, to the best of our knowledge, forgotten.

Meanwhile, the theory of Lie groupoids was created by Ehresmann during the 1950s, and their infinitesimal counterpart, the Lie algebroids, were introduced by Pradines in 1967 [35]. Groupoid theory has been developed by many authors, in the context of homotopy theory and $C^{*}$-algebras, as well as in differential geometry, and it has come to differ in substantial respects from the elementary theory of groups. However, there is a Lie functor which associates to a Lie groupoid, $G$, a Lie algebroid, denoted $A G$, by a construction which extends in a straightforward way the construction of the Lie algebra of a Lie group. The resulting Lie theory provides a very broad framework which encompasses many of the infinitesimal constructions of differential geometry.

The concept of action of a Lie groupoid on a fibered manifold goes back to Ehresmann [8], who showed that when a groupoid acts on a space that is fibered over the base of the groupoid, the pullback of the groupoid by the fibration map is, in a natural way, a groupoid, called the action groupoid or transformation groupoid. The development of an abstract theory of Lie algebroids is more recent: in $\mathbf{1 7}$ Higgins and Mackenzie provided a basic set of algebraic constructions for abstract Lie algebroids modelled on those known in groupoid theory. In particular, they defined the infinitesimal action of a Lie algebroid on a fibered manifold and the associated action Lie algebroid or transformation Lie algebroid. The Lie algebroid of an action Lie groupoid is the action Lie algebroid of the corresponding infinitesimal action; however the action Lie algebroid of an infinitesimal Lie algebra action may integrate to a Lie groupoid which is not necessarily an action groupoid. This may at first seem to be a negative feature, but in fact the groupoid in such a situation provides a global object encoding the infinitesimal action even though a global action is not available. Here, as is generally the case with abstract Lie algebroids, determining the conditions under which a particular construction for Lie algebroids will admit a global analogue is a difficult problem. See Dazord [7], and Moerdijk and Mrčun 33 for a systematic account of the integration of Lie algebroid actions which includes many applications.

The aim of this paper is to relate the concept of Lie algebroid action to the earlier work [22] on representations by derivative endomorphisms (and, correspondingly, Lie groupoid actions and representations by semi-linear isomorphisms.) There are several aspects to the relationship. First, the derivative endomorphisms of the space of sections of a vector bundle are the sections of a Lie algebroid (Theorem 1.4). In [30, Mackenzie called this Lie algebroid the Lie algebroid of covariant differential operators, and denoted it by $\operatorname{CDO}(E)$, for a vector bundle $E$. Here, we denote it by $\mathfrak{D}(E)$. A derivative representation of a Lie algebra on a vector bundle is defined as a morphism into the Lie algebra of sections of $\mathfrak{D}(E)$, while a representation of a Lie algebroid on a vector bundle is a morphism into this Lie 
algebroid. We prove that the derivative representations of Lie algebras on vector bundles coincide with the representations of action Lie algebroids (Theorem 2.4). Then, with a suitable definition of the derivative representations of a Lie algebroid on a vector bundle, we show that this one-to-one correspondence can be extended to the case of Lie algebroid actions (Theorem 3.5).

In Sections 4 and 5 , we present global versions of the preceding results. We define the semi-linear isomorphisms of the space of sections of a vector bundle. A semi-linear representation of a Lie group on a vector bundle is defined as a morphism into the group of semi-linear isomorphisms of its space of sections, satisfying a smoothness condition, while a representation of a Lie groupoid on a vector bundle is a morphism of Lie groupoids into the Lie groupoid of all linear isomorphisms from a fiber to some (in general different) fiber of the bundle. In Theorem 4.8 we prove that the semi-linear representations of Lie groups on vector bundles coincide with the representations of their action groupoids. For a groupoid acting on a fibered manifold, $F$, there is likewise an action groupoid; considering an action of the groupoid on a vector bundle with base $F$, we show in Proposition 5.5 that there is an associated semi-linear representation which is a group representation of the group of bisections of the groupoid.

In the Appendix, we recall the definition of the twisted derivations of an algebra, and we show that the pseudo-linear endomorphisms of the module of sections $\Gamma E$ of a vector bundle $E$ with base $M$ can be defined in terms of twisted derivations of an algebra whose underlying vector space is $C^{\infty}(M) \oplus \Gamma E$. In particular, the derivative endomorphisms can be defined in terms of the derivations of this algebra. Similarly, the semi-linear isomorphisms can be defined in terms of algebra automorphisms.

Throughout the paper, the manifolds that we consider are assumed to be smooth and second countable, and all maps are assumed to be smooth.

Acknowledgments. The authors would like to extend their warmest thanks to Mike Prest and Ted Voronov, the organizers of the workshop on "Quantization, deformations and new homological and categorical methods in mathematical physics" (Manchester, 2001) which was particularly rich in stimulating lectures. Although the present paper does not reflect the contents of the authors' lectures in Manchester, it was the workshop that made its realization possible.

\section{Infinitesimal automorphisms of vector bundles}

Consider a vector bundle $(E, q, M)$ and let $C^{\infty}(M)$ be the algebra of $\mathbb{R}$-valued functions on $M$. We are concerned with first-order differential operators, $D: \Gamma E \rightarrow$ $\Gamma E$, for which there exists a vector field $D_{M}$ on $M$ such that

$$
D(f \psi)=f D(\psi)+D_{M}(f) \psi,
$$

for all $f \in C^{\infty}(M)$ and all $\psi \in \Gamma E$. Below we explain that operators on modules over a ring satisfying (11), or some variant of it, have been studied under a variety of names. In the case of the module of sections of a vector bundle, we shall call such a differential operator a derivative endomorphism of $\Gamma E$.

Definition 1.1. Let $(E, q, M)$ be a vector bundle. A derivative endomorphism of $\Gamma E$ is an $\mathbb{R}$-linear endomorphism $D$ of $\Gamma E$ such that there exists an $\mathbb{R}$-linear endomorphism, $D_{M}$, of $C^{\infty}(M)$ satisfying (1D). 
It follows that $D$ is a first-order differential operator. It also follows that $D_{M}$ is a derivation of $C^{\infty}(M)$, and therefore corresponds to a vector field on the base manifold $M$. The differential operator $D$ is of order 0 if and only if $D_{M}=0$.

EXAMPLE 1.2. Given any linear connection $\nabla$ in a vector bundle $E$, and any vector field $X$ on the base $M$, the covariant derivation $\nabla_{X}$ satisfies (1).

Recall that the symbol, $\sigma(D)$, of a first-order differential operator $D$ is defined by

$$
\sigma(D)(d f)=[D, f]
$$

where $f$ is any smooth function on $M$ and $[D, f]$ is the commutator of the operators $D$ and multiplication by $f$. Condition (1i) is equivalent to the requirement that the symbol of $D$, evaluated at any 1 -form $\xi$ on $M$ at $x \in M$, be a scalar multiple of the identity of the fiber $E_{x}$ at $x, \sigma(D) \xi=\left\langle\xi, D_{M}\right\rangle \operatorname{Id}_{E_{x}}$. Therefore, a first-order differential operator is a derivative endomorphism of $\Gamma E$ if and only if it has scalar symbol.

Whereas the first-order differential operators on a vector bundle of rank $>1$ do not constitute a Lie algebra under the commutator, a simple computation shows that the commutator

$$
\left[D_{1}, D_{2}\right]=D_{1} \circ D_{2}-D_{2} \circ D_{1}
$$

of two derivative endomorphisms is also a derivative endomorphism, and that

$$
\left[D_{1}, D_{2}\right]_{M}=\left[\left(D_{1}\right)_{M},\left(D_{2}\right)_{M}\right] .
$$

It is also clear that the derivative endomorphisms form a module over $C^{\infty}(M)$. The $\mathbb{R}$-Lie algebra structure and the $C^{\infty}(M)$-module structure on the vector space of derivative endomorphisms of $E$ interact according to a Leibniz rule,

$$
\left[D_{1}, f D_{2}\right]=f\left[D_{1}, D_{2}\right]+\left(D_{1}\right)_{M}(f) D_{2}
$$

which is a property characteristic of the sections of a Lie algebroid. We recall the concept of Lie algebroid, introduced by Pradines in [35].

Definition 1.3. Let $M$ be a manifold. A Lie algebroid on $M$ is a vector bundle $(A, q, M)$ together with a vector bundle map $a: A \rightarrow T M$ over $M$, called the anchor of $A$, and a bracket [, ]: $\Gamma A \times \Gamma A \rightarrow \Gamma A$ which is $\mathbb{R}$-bilinear and alternating, satisfies the Jacobi identity, and is such that

$$
\begin{gathered}
a([X, Y])=[a(X), a(Y)], \\
{[X, f Y]=f[X, Y]+a(X)(f) Y,}
\end{gathered}
$$

for all $X, Y \in \Gamma A, f \in C^{\infty}(M)$.

Given Lie algebroids $A$ and $A^{\prime}$ on the same base $M$, a Lie algebroid morphism from $A$ to $A^{\prime}$ is a vector bundle morphism $\varphi: A \rightarrow A^{\prime}$ over $M$ such that

$$
\begin{gathered}
a^{\prime} \circ \varphi=a, \\
\varphi([X, Y])=[\varphi(X), \varphi(Y)],
\end{gathered}
$$

for all $X, Y \in \Gamma A$.

For Lie algebroids $A$ and $A^{\prime}$ on different base manifolds, the notion of morphism is considerably more complicated; see [17].

Any Lie algebra is a Lie algebroid over $M=\{\cdot\}$, and for any manifold $M$, the tangent bundle $T M$ is a Lie algebroid with anchor $a=\operatorname{Id}_{T M}$. Further examples will be introduced below. 
THEOREM 1.4. Given a vector bundle $E$ on base $M$, there is a Lie algebroid $\mathfrak{D}(E)$ on $M$, the smooth sections of which are the derivative endomorphisms of $\Gamma E$, for which the bracket is the commutator bracket (2) and the anchor is the map $D \mapsto D_{M}$.

Proof. The first-order differential operators are the sections of a vector bundle $\operatorname{Diff}^{1}(E)$ on $M$. The symbol map is a vector bundle morphism, $\sigma: \operatorname{Diff}^{1}(E) \rightarrow$ $\operatorname{Hom}\left(T^{*} M, \operatorname{End}(E)\right)$, where $\operatorname{End}(E)$ is the vector bundle over $M$ the fibers of which are the endomorphisms of the fibers of $E$. It is a surjective submersion and has as its kernel the differential operators of order 0 . There is thus a short exact sequence of vector bundles over $M$,

$$
\operatorname{End}(E) \stackrel{\subseteq}{\longrightarrow} \operatorname{Diff}^{1}(E) \stackrel{\sigma}{\longrightarrow} \operatorname{Hom}\left(T^{*}(M), \operatorname{End}(E)\right) .
$$

Identifying $\operatorname{Hom}\left(T^{*}(M), \operatorname{End}(E)\right)$ with $T M \otimes \operatorname{End}(E)$ in the canonical way, we define $\mathfrak{D}(E)$ to be the pullback vector bundle defined by the symbol map and the injection $T M \rightarrow T M \otimes \operatorname{End}(E), X \mapsto X \otimes \mathrm{I} d_{E}$, according to the diagram:

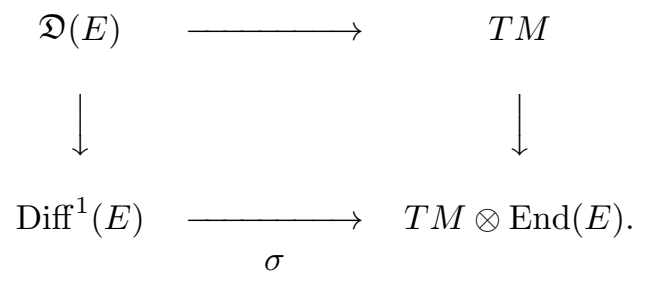

The pullback exists because $\sigma$ is a surjective submersion. Since the right-hand vertical arrow is an injective immersion, it follows that so is the left-hand arrow, and we can therefore regard $\mathfrak{D}(E)$ as a vector subbundle of $\operatorname{Diff}^{1}(E)$. Similarly, because $\sigma$ is a surjective submersion, it follows that so is the top arrow, which we denote by $a$. Clearly the kernel of $a$ is still End(E), and there is an exact sequence of vector bundles over $M$,

$$
\operatorname{End}(E)>\longrightarrow \mathfrak{D}(E) \stackrel{a}{\longrightarrow} T M
$$

where the sections of $\mathfrak{D}(E)$ are those first-order differential operators $D: \Gamma E \rightarrow \Gamma E$ for which there exists a vector field $D_{M}=a(D)$ on $M$ such that (11) is satisfied.

We have already observed that for any pair of derivative endomorphisms $D_{1}, D_{2}$ in $\Gamma E$, the bracket (2) is also a derivative endomorphism of $\Gamma E$, with $a\left(\left[D_{1}, D_{2}\right]\right)=$ $\left[a\left(D_{1}\right), a\left(D_{2}\right)\right]$, and that (任) is satisfied. Thus the proof that $\mathfrak{D}(E)$ is a Lie algebroid on $M$ with anchor $a$ is complete.

In general, a Lie algebroid is called transitive if the anchor is surjective. It was shown in the course of the proof of Theorem 1.4 that $\mathfrak{D}(E)$ is transitive. A rightinverse $\nabla: T M \rightarrow \mathfrak{D}(E)$ to the anchor is a linear connection in $E$ in the standard sense. The curvature $R_{\nabla}: T M \oplus T M \rightarrow \operatorname{End}(E)$ of $\nabla$ is given by

$$
R_{\nabla}\left(X_{1}, X_{2}\right)=\nabla_{\left[X_{1}, X_{2}\right]}-\left[\nabla_{X_{1}}, \nabla_{X_{2}}\right] .
$$

In particular, the connection is flat if and only if $\nabla$ is a morphism of Lie algebroids.

Now consider the case of a trivial vector bundle, $E=M \times V$, and define a morphism from the vector bundle $T M \oplus(M \times \mathfrak{g l}(V))$ into $\mathfrak{D}(E)$ by

$$
(X+u)(\psi)=X(\psi)+u(\psi): M \rightarrow V
$$


where $X(\psi)$ is the Lie derivative. For an arbitrary vector bundle, such a direct sum decomposition of $\mathfrak{D}(E)$ is given locally by a trivialization of $E$, while a global decomposition is defined by the choice of a linear connection, $\nabla$, on $E$. Let $\nabla$ also denote the extension of the chosen connection to the sections of $\operatorname{End}(E)$. Then for $D_{1}=X_{1}+u_{1}$ and $D_{2}=X_{2}+u_{2}$,

$$
\left[D_{1}, D_{2}\right]=\left[X_{1}, X_{2}\right]+\left(\nabla_{X_{1}} u_{2}-\nabla_{X_{2}} u_{1}+\left[u_{1}, u_{2}\right]-R_{\nabla}\left(X_{1}, X_{2}\right)\right) .
$$

By definition, when $R_{\nabla}=0$, this bracket gives $T M \oplus \operatorname{End}(E)$ the trivial Lie algebroid structure. Thus the Lie algebroid $\mathfrak{D}(E)$ is trivial if and only if $E$ admits a flat connection.

The following result follows from a theorem of Bourbaki [4, III.10.9, Prop. 14].

Proposition 1.5. The derivative endomorphisms of $\Gamma E$ are the $\mathbb{R}$-linear endomorphisms of $\Gamma E$ which can be extended to derivations of the algebra of sections of the tensor algebra of $E$.

The notion of derivative endomorphism is in essence purely algebraic. Given a left module $\mathcal{E}$ over a ring $\mathcal{C}$, a derivation of $\mathcal{E}$ over a derivation $D_{\mathcal{C}}$ of $\mathcal{C}$ is an additive map $D: \mathcal{E} \rightarrow \mathcal{E}$ such that

$$
D(f \psi)=f D(\psi)+D_{\mathcal{C}}(f) \psi
$$

for all $f \in \mathcal{C}$ and $\psi \in \mathcal{E}$. We can also refer to a derivation of $\mathcal{E}$ over a derivation $D_{\mathcal{C}}$ of $\mathcal{C}$ as a derivative endomorphism of $\mathcal{E}$. Note, however, that in the general case, $D$ does not determine $D_{\mathcal{C}}$.

Bourbaki [4] defines a very general notion of derivation (involving six possibly different modules over a ring, three bilinear maps and three linear maps), and shows that the set of those derivations that belong to the "cas I" is closed under the commutator. The definition of the derivations in "cas I" includes the derivative endomorphisms as a particular case. The derivative endomorphisms of $\mathcal{E}$ are also a special case of the pseudo-linear endomorphisms, introduced and studied in the case of a finitely generated module over a ring by Jacobson in [19]. In fact, Jacobson uses the term 'vector space over a field' for module over a ring and the term transformation for endomorphism. His definition of the pseudo-linear transformations involves an automorphism $f \mapsto \bar{f}$ of $\mathcal{C}$ such that the following generalization of (10) is satisfied,

$$
D(f \psi)=\bar{f} D(\psi)+D_{\mathcal{C}}(f) \psi .
$$

This generalization is applicable, for instance, when one considers modules over rings of complex-valued functions, in which case $f \mapsto \bar{f}$ is the conjugation of complex numbers. Jacobson gave what amounts to the covariant derivation of vector fields in $\mathbb{R}^{n}$ as an example of such transformations, and derived the transformation law for connections under a change of basis. Then he studied the generalization of the theory of elementary divisors to the case of these pseudo-linear transformations. In 20], he called differential transformations those pseudo-linear transformations for which $f=\bar{f}$.

Working in this algebraic setting, Herz [15, 16], referring to Jacobson, showed that the differential transformations of a vector space over a commutative field form a Lie pseudo-algebra, a notion which he introduced. This may now be seen as an algebraic form of the notion of Lie algebroid in which vector bundles over manifolds are replaced by modules over rings, vector fields by derivations of rings, and so on. 
Herz studied the sub-Lie-pseudo-algebras of a Lie pseudo-algebra over a skew field, and then showed that what he called the kernel of the Lie pseudo-algebra is a Lie algebra. (There is a corresponding result in the theory of Lie algebroids, that the kernel of the anchor in a transitive Lie algebroid is a Lie algebra bundle. However, the algebraic results proved for Lie pseudo-algebras do not automatically imply the corresponding theorems in the category of Lie algebroids, since submodules of finitely generated projective modules do not necessarily correspond to vector subbundles.)

A Lie pseudo-algebra that generalizes the Lie algebroid $\mathfrak{D}(E)$ to the more general setting of modules over Lie pseudo-algebras was constructed in $[\mathbf{2 9}]$; the construction is also given in [18]. For references dealing with Lie pseudo-algebras, also now known as Lie-Rinehart algebras, see 31 .

While the algebraic properties of covariant derivatives were already stated explicitly by Schouten in 1924, in his book, Ricci Kalkül, the algebraization of the theory of connections is due to Koszul [25, and a very general formalization was later accomplished by a student of Ehresmann, C. M. de Barros, in his thesis [2]. There, he introduced the translations infinitésimales graduées, of which the derivative endomorphisms are a particular case, and he studied the représentations infinitésimales graduées, which generalize the lois de dérivation of Koszul. (We shall deal with such Lie algebra representations in the next section.) When the module being considered is that of the sections of a vector bundle, the derivative endomorphisms and their higher-order analogues were introduced by Palais 34 under the name quasi-scalar differential operators in his analysis of elliptic selfadjoint operators on Riemannian vector bundles. At about the same time, Ngô Van Que [36], 37], 38 used operators satisfying (11) in his work on Lie groupoids. In [22], the name derivative endomorphisms of $\Gamma E$ for operators satisfying (11) was introduced, and it was remarked that such operators are the first-order differential operators on $E$ with scalar symbol. Such operators were independently introduced in [30], 31] where, because of the important example 1.2, they are called covariant differential operators. A number of papers [3], [39] introduce an Atiyah algebra of a vector bundle $E$, which is again the module of sections of $\mathfrak{D}(E)$.

Derivative endomorphisms arise whenever the infinitesimal of a representation of a group defined by an action on a vector bundle is considered. In [14], Hermann introduced the first-order differential operators satisfying relation (1) and he briefly indicated "the geometric genesis of these operators". In [22], the semilinear endomorphisms of $\Gamma E$ were introduced (see Section 1 ) and it was shown that an action of a Lie group on the sections of a vector bundle by semi-linear transformations differentiates to an action of its Lie algebra by differential operators which are derivative endomorphisms. It was stated (p. 86) that the Lie algebra of derivative endomorphisms of $\Gamma E$ is to be considered "as the Lie algebra of the 'infinite-dimensional Lie group' of all automorphisms of the vector bundle $E$ ". One may say that a derivative endomorphism of $\Gamma E$ is an infinitesimal automorphism of the vector bundle $E$, in the same way that a vector field is an infinitesimal automorphism of a manifold. Derivative endomorphisms play a crucial role throughout the theory of Lie algebroids; it was argued in detail in [30] that $\mathfrak{D}(E)$ is the correct generalization to vector bundles of the general linear Lie algebra $\mathfrak{g l}(V)$ of a vector space $V$. Theorem 1.4 was proved in [30]. We will discuss the relationships between these approaches further below. 


\section{Derivative representations of Lie algebras and action Lie algebroids}

We again consider a vector bundle $(E, q, M)$. Since the derivative endomorphisms of $\Gamma E$ play the role of the infinitesimal automorphisms of the vector bundle $E$, it is natural to define:

DeFinition 2.1. A derivative representation of a Lie algebra $\mathfrak{g}$ on $E$ is a morphism of $\mathfrak{g}$ into the Lie algebra $\Gamma \mathfrak{D}(E)$.

Associated to a derivative representation, $\rho$, of $\mathfrak{g}$ on $E$, there is an infinitesimal action of $\mathfrak{g}$ on $E, X \mapsto(\rho(X))_{M}$. Of course, if the base $M$ of $E$ is a point, we recover the usual notion of linear representation.

EXAMPLE 2.2. Let us consider invariant prequantization as defined in 13. Let $(M, \omega)$ be a symplectic manifold, and suppose that $\omega$ is integral. Then there is a complex line bundle $E$ on $M$, together with a Hermitian fiber-metric, and a connection $\nabla$ in $E$ with respect to which the metric is parallel, and such that the curvature $R_{\nabla}$ of $\nabla$ satisfies

$$
R_{\nabla}=-i \omega
$$

The prequantization action of $C^{\infty}(M)$ on sections of $E$ is the map, $\delta: C^{\infty}(M) \rightarrow$ $\Gamma \mathfrak{D}(E)$, defined by

$$
\delta(f)(\psi)=\nabla_{X_{f}}(\psi)+i f \psi,
$$

where $f \in C^{\infty}(M), \psi \in \Gamma E$, and where $X_{f}$ is the Hamiltonian vector field of $f$. It maps the Poisson bracket to the Lie algebroid bracket of $\mathfrak{D}(E)$, respects the anchors in the sense that $a(\delta(f))=X_{f}$ for $f \in C^{\infty}(M)$, and takes values in the Lie subalgebroid $\mathfrak{D}_{0}(E)$ of $\mathfrak{D}(E)$, whose sections respect the metric.

Now suppose that $G \times M \rightarrow M$ is a Hamiltonian action of a Lie group $G$ on $M$, with a bracket-preserving moment map, $\widehat{J}: \mathfrak{g} \rightarrow C^{\infty}(M)$. Then $X_{\widehat{J}(X)}=X_{M}$, for all $X \in \mathfrak{g}$, where $X \mapsto X_{M}, \mathfrak{g} \rightarrow \Gamma T M$, is the infinitesimal action. Now $\rho=\delta \circ \widehat{J}: \mathfrak{g} \rightarrow \Gamma \mathfrak{D}(E)$ is a derivative representation of $\mathfrak{g}$ on $E$.

There is also a well-known notion of a Lie algebroid representation, which we recall.

Definition 2.3. A representation of a Lie algebroid $A$, also on base $M$, on the vector bundle $E$ is a Lie algebroid morphism from $A$ to $\mathfrak{D}(E)$.

If the base $M$ is a point, $A$ is merely a Lie algebra and $E$ is a vector space, and this definition reduces to that of a linear representation.

To express the relationship between these notions, we recall the definition of an action Lie algebroid. Consider an infinitesimal action of a Lie algebra $\mathfrak{g}$ on a manifold $M, i$. e., a map $X \mapsto X_{M}, \mathfrak{g} \rightarrow \Gamma T M$, which is $\mathbb{R}$-linear, and preserves brackets, $[X, Y]_{M}=\left[X_{M}, Y_{M}\right]$ for all $X, Y \in \mathfrak{g}$. Extend this notation to maps $V: M \rightarrow \mathfrak{g}$ so that $V_{M}$ is the vector field on $M$ defined by $V_{M}(m)=\left(V(m)_{M}\right)(m)$, for $m \in M$. Then the trivial vector bundle $M \times \mathfrak{g}$ on $M$ acquires a Lie algebroid structure with anchor $a: M \times \mathfrak{g} \rightarrow T M$ defined by $a(m, X)=X_{M}(m)$, and bracket

$$
[V, W]=V_{M}(W)-W_{M}(V)+[V, W]^{\bullet},
$$

where $V_{M}(W)$ denotes the Lie derivative with respect to $V_{M}$ of the vector-valued function $W$, and $[V, W]^{\bullet}$ is the pointwise bracket of maps into $\mathfrak{g}$. With this Lie algebroid structure, the trivial vector bundle $M \times \mathfrak{g}$ is called the action Lie algebroid corresponding to the infinitesimal action $X \mapsto X_{M}$, and we denote it by $\mathfrak{g} \varangle M$. 
Observe that if $V, W: M \rightarrow \mathfrak{g}$ are constant maps, then the Lie algebroid bracket $[V, W]$ is constant also, and is constant at the bracket in $\mathfrak{g}$ of the values of $V$ and $W$. In fact this property, together with the anchor $a$ : $\mathfrak{g} \varangle M \rightarrow T M$ and (6), determines (11).

We can now relate the two concepts in Definitions 2.1 and 2.3.

THEOREM 2.4. Let $X \mapsto X_{M}$ be an infinitesimal action of a Lie algebra $\mathfrak{g}$ on $M$. There is a one-to-one correspondence between derivative representations of $\mathfrak{g}$ on $E$, associated to the given infinitesimal action, and representations of the action Lie algebroid $\mathfrak{g} \varangle M$ on $E$.

Proof. Let $\rho: \mathfrak{g} \rightarrow \Gamma \mathfrak{D}(E)$ be a derivative representation of $\mathfrak{g}$ associated to the given infinitesimal action $X \mapsto X_{M}$. By definition, the anchor of $\rho(X)$ is $X_{M}$. We define a vector bundle morphism $\sigma$ from $M \times \mathfrak{g}$ to $\mathfrak{D}(E)$ by

$$
\sigma(m, X)=(\rho(X))(m) .
$$

This vector bundle morphism is a morphism of Lie algebroids from $\mathfrak{g} \varangle M$ to $\mathfrak{D}(E)$ because (i) it clearly satisfies the condition on the anchors, and (ii) the bracket condition is satisfied for constant maps from $M$ to $\mathfrak{g}$. Conversely, if $\sigma$ is a representation of $\mathfrak{g} \varangle M$ on $E$, formula 12 defines a morphism $\rho$ from $\mathfrak{g}$ to $\Gamma \mathfrak{D}(E)$.

The significance of Theorem 2.4 is that, on the one hand, it can be immediately generalized once we extend the definition of action Lie algebroid to the case of Lie algebroids that are not Lie algebras, and on the other hand, when the given actions globalize, it admits a global formulation. We deal with these two aspects in the following sections.

ExAMPLE 2.5. If $\rho$ is the map defined in Example 2.2, the map $\sigma$ defined by (12) is a representation of $\mathfrak{g} \varangle M$ on $E$, more precisely, a morphism of Lie algebroids from $\mathfrak{g} \varangle M$ to the Lie subalgebroid $\mathfrak{D}_{0}(E)$ of $\mathfrak{D}(E)$.

ExAMPLE 2.6. Given any principal bundle $P(M, H)$ and a linear representation of $H$ on a vector space $V$, let $E=P \times_{H} V$ be the associated vector bundle. Let $A=T P / H$ be the Atiyah Lie algebroid of the principal bundle. There is a representation $\sigma$ of $A$ on $E$ defined as follows (see, e.g., 30, App.A]). A section $\psi$ of $E$ can be identified with an $H$-equivariant map, $\widetilde{\psi}: P \rightarrow V$, and a section $X$ of $T P / H$ can be identified with an $H$-invariant vector field $\widetilde{X}$ on $P$. Since the Lie derivative of $\widetilde{\psi}$ with respect to $\widetilde{X}$ is also an $H$-equivariant map from $P$ to $V$, it corresponds to a section $\sigma(X)(\psi)$.

If, in particular, $P$ is a Lie group $G$ with $H$ a closed subgroup, this construction associates to any linear representation of $H$ on $V$ the representation $\sigma$ of $T G / H$ on $E=G \times_{H} V$. On the other hand, the Lie algebroid $T G / H$ is canonically isomorphic to the action Lie algebroid $\mathfrak{g} \varangle(G / H)$ arising from the standard action of $\mathfrak{g}$ on $M=G / H$, under the map $T G / H \rightarrow \mathfrak{g} \varangle(G / H)$ defined by the canonical right identificatiion of $T G$ with $G \times \mathfrak{g}$. It follows by Theorem 2.4 that there is a one-to-one correspondence between the linear representations of $\mathfrak{h}$ on $V$ and the derivative representations of $\mathfrak{g}$ on $E=G \times{ }_{H} V$.

In this case, the derivative representation of $\mathfrak{g}$ on $E=G \times{ }_{H} V$ associated to the representation $\sigma$ of $A=T G / H$ on $E$ is the infinitesimal of the linear representation of $G$ induced from that of $H$. So this result is the infinitesimal counterpart of an 
observation that was made in 22] relating semi-linear representations of Lie groups to induced representations.

ExAmple 2.7. A representation of a Lie algebroid $A$ on a vector bundle $E$ extends to a representation by derivations of its exterior algebra, that is, a morphism from the $\mathbb{R}$-Lie algebra $\Gamma A$ to the $\mathbb{R}$-Lie algebra of derivations of $\Gamma(\bigwedge E)$, and conversely the restriction to $\Gamma E$ of such a representation by derivations of $\Gamma(\bigwedge E)$ is a representaion of $A$ on $E$ in the sense of Definition 2.3.

Remark 2.8. Consider a Lie algebroid $A$ and a vector bundle $E$ on the same base $M$. Several authors [40 [10] consider the $A$-connections on $E$, defined as the vector bundle morphisms $A \rightarrow \mathfrak{D}(E)$ satisfying condition (7) on the anchors, without a bracket condition. If $A=T M$, this is precisely the standard concept of linear connection in $E$.

The curvature of such an $A$-connection $\nabla$ is defined as in (9). Now a representation of $A$ on $E$ is precisely a flat $A$-connection on $E$. Extensive use of the notion of $A$-connection and of representation of Lie algebroids was made in 40 [10], especially the representations of $A$ on $\wedge^{n} A$ where $n$ is the rank of $A$. The one-to-one correspondence described in the proof of Theorem 2.4 is actually the restriction to the derivative endomorphisms of a one-to-one correspondence between derivative pre-representations of $\mathfrak{g}$ on $E$, where we only require linearity of the map $\mathfrak{g} \rightarrow \Gamma \mathfrak{D}(E)$, and $A$-connections on $E$.

The vector bundle that we have called the Atiyah Lie algebroid of a principal bundle was introduced by Atiyah in 1957 [1], ten years before Pradines introduced the abstract concept of Lie algebroid. It is also called the gauge algebroid of the principal bundle, and its sections are then called infinitesimal gauge transformations of the bundle. The definition of derivative representation comes from [22]. The derivative representations are a particular case of the graded infinitesimal representations of de Barros [2]. When $\mathfrak{g}$ is the Lie algebra of derivations of $C^{\infty}(M)$ and when the map $\mathfrak{g} \rightarrow \Gamma \mathfrak{D}(E)$ is $C^{\infty}(M)$-linear, a derivative representation is a flat derivation law in the sense of Koszul [25]. The definition of Lie algebroid representation may be found in [30, III.2.9]; it was probably first formulated in [38]. The corresponding algebraic notion is that of a left module over a Lie pseudo-algebra.

\section{Lie algebroid actions and representations}

In this section we first consider the infinitesimal actions of a Lie algebroid $A$ with base $M$ on a fibered manifold with base $M$, that is, on a surjective submersion onto $M$. Such an infinitesimal action of $A \rightarrow M$ on $\varphi: F \rightarrow M$ defines a Lie algebroid structure on the vector bundle $\varphi^{*} A$, the pullback of $A$, which is called the action Lie algebroid defined by the given infinitesimal action of $A$ on $F$.

We then extend the definition of the derivative representations to the case of a Lie algebroid $A$ and a vector bundle with base $F$. As a particular case of this notion, we recover Definition 2.1 in the case where $M$ is a point. Finally in this section we show that the one-to-one correspondence between derivative representations and representations of action Lie algebroids can be extended to this more general situation.

Definition 3.1. Let $A$ be a Lie algebroid on $M$, and let $\varphi: F \rightarrow M$ be a fibered manifold. An infinitesimal action of $A$ on $F$ is an $\mathbb{R}$-linear map $X \mapsto$ $X_{F}, \Gamma A \rightarrow \Gamma T F$, such that 
(i) for each $X \in \Gamma A, X_{F}$ is projectable to $a(X)$,

(ii) the map $X \mapsto X_{F}$ preserves brackets,

(iii) the map $X \mapsto X_{F}$ is $C^{\infty}(M)$-linear in the following sense: for each $f \in C^{\infty}(M)$ and each $X \in \Gamma A$,

$$
(f X)_{F}=(f \circ \varphi) X_{F} .
$$

If $\Gamma T F$ is given the $C^{\infty}(M)$-module structure defined by $(f, Y) \mapsto(f \circ \varphi) Y$, $C^{\infty}(M) \times \Gamma T F \rightarrow \Gamma T F$, then Equation (13) is the condition that $X \mapsto X_{F}$ is a morphism of left modules.

If, in particular, the base $M$ is a point, then $A$ is a Lie algebra, and we recover the notion of infinitesimal action of a Lie algebra on a manifold $F$, conditions (i) and (iii) being trivially satisfied.

If $F=M$ and $\varphi$ is the identity, an infinitesimal action of the Lie algebroid $A$ on the manifold $M$ is a $C^{\infty}(M)$-linear map $X \mapsto X_{M}$ from $\Gamma A$ to $\Gamma T M$, i.e., a morphism of vector bundles from $A$ to $T M$, which by condition (i) is necessarily the anchor of $A$.

It is shown in [17] that whenever an infinitesimal action of $A$ on $\varphi: F \rightarrow M$ is defined, there is an associated Lie algebroid structure on the pullback vector bundle, $\varphi^{*} A$,

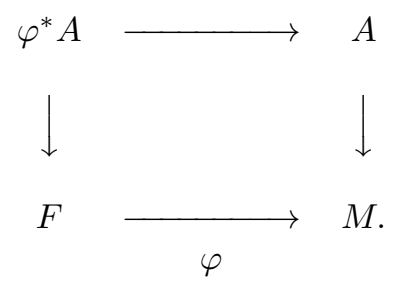

With this Lie algebroid structure, $\varphi^{*} A$ is called the action Lie algebroid associated to the infinitesimal action $X \mapsto X_{F}$, and it is denoted $A \varangle F$. We recall the definition of this Lie algebroid structure. We identify the module of sections of the pullback with $C^{\infty}(F) \otimes \Gamma A$, where the tensor product is over $C^{\infty}(M)$. Thus $h \otimes f X=h(f \circ \varphi) \otimes X$ and $h \otimes X$ is identified with $h(X \circ \varphi)$, for $h \in C^{\infty}(F), f \in$ $C^{\infty}(M), X \in \Gamma A$. Then $\varphi^{*} A$ is a Lie algebroid with the following anchor, $a_{F}$,

$$
a_{F}(h \otimes X)=h X_{F},
$$

and $\mathbb{R}$-bilinear bracket,

$$
[h \otimes X, k \otimes Y]=h k \otimes[X, Y]+h X_{F}(k) \otimes Y-k Y_{F}(h) \otimes X,
$$

where $h, k \in C^{\infty}(F), X, Y \in \Gamma A$.

If the base $M$ is a point, this Lie algebroid over $F$ is merely the product $F \times A$ with the action Lie algebroid structure over base $F$ defined in Section 2.

The notion of action and the construction of an action Lie algebroid extend to the situation in which $\varphi$ is an arbitrary smooth map, but we shall not need this generality here.

ExAmPle 3.2. Let $A$ be any Lie algebroid on a connected base $M$, and let $f: \widetilde{M} \rightarrow M$ be any covering, with group $\pi$. Then there is a canonical infinitesimal action of $A$ on $\widetilde{M}$ in which $X_{\widetilde{M}}$ is the $\pi$-invariant lift of $a(X)$ to $\widetilde{M}$.

Example 3.3. Given any principal bundle $P(M, H)$, the Atiyah Lie algebroid $T P / H$ with base $M$ acts on $P \rightarrow M$ by $X \mapsto \vec{X}$, where $\vec{X}$ is the right-invariant 
vector field on $P$ defined by $X \in \Gamma(T P / H)$; the action Lie algebroid is canonically isomorphic to $T P$.

In view of Theorem 2.4, we shall define the notion of derivative representation of a Lie algebroid $A$ with base $M$ on a vector bundle $E$ with base $F$, associated to an infinitesimal action of $A$ on $F$, in such a way that the one-to-one correspondence between derivative representations and representations of the action Lie algebroid is extended.

Definition 3.4. Let $A$ be a Lie algebroid on $M$, and let $\varphi: F \rightarrow M$ be a fibered manifold. Let $X \mapsto X_{F}, \Gamma A \rightarrow \Gamma T F$, be an infinitesimal action of $A$ on $F$. Let us consider a vector bundle $E$ on $F$. A derivative representation of $A$ on $E$ associated to the infinitesimal action $X \mapsto X_{F}$ is a morphism, $\rho$, of Lie algebras from $\Gamma A$ to $\Gamma \mathfrak{D}(E)$ such that

(i) for any $X \in \Gamma A$,

$$
(\rho(X))_{F}=X_{F},
$$

(ii) $\rho$ is $C^{\infty}(M)$-linear in the following sense: for each $f \in C^{\infty}(M)$ and each $X \in \Gamma A$,

$$
\rho(f X)=(f \circ \varphi) \rho(X) .
$$

Since for any $X \in \Gamma A, \rho(X)$ is a section of $\mathfrak{D}(E)$, condition (16) expresses the fact that, for any $h \in C^{\infty}(F), \psi \in \Gamma E$,

$$
\rho(X)(h \psi)=h \rho(X)(\psi)+X_{F}(h) \psi .
$$

Theorem 3.5. Let $A, F, X \mapsto X_{F}$ and $E$ be as in Definition 3.4. There is a one-to-one correspondence between derivative representations of $A$ on $E$ associated to the infinitesimal action $X \mapsto X_{F}$ and representations of the action Lie algebroid $A \varangle F$ on $E$.

Proof. We sketch the proof which is very similar to that of Theorem 2.4. If $\rho: \Gamma A \rightarrow \Gamma \mathfrak{D}(E)$ is a derivative representation of $A$ on $E$ associated to the given infinitesimal action of $A$ on $F$, we define $\sigma: \varphi^{*} A \rightarrow E$ in terms of sections of the form $h \otimes X, h \in C^{\infty}(F), X \in \Gamma A$, by

$$
\sigma(h \otimes X)(p)=h(p) \rho(X)(p),
$$

for any $p \in F$. Then $\sigma$ is a representation of the Lie algebroid $\varphi^{*} A$ on $E$.

Conversely, if $\sigma$ is a representation of the Lie algebroid $\varphi^{*} A$ on $E$, we set, for $X \in \Gamma A$, and $p \in F$,

$$
\rho(X)(p)=\sigma(X \circ \varphi)(p) .
$$

Then $\rho$ is a derivative representation of $A$ on $E$.

Thus we have obtained a generalization of Theorem 2.4 to the case of Lie algebroids acting on vector bundles.

The Lie algebroid structure (15) of an action Lie algebroid corresponds in the algebraic setting treated by Fel'dman [11] to a crossed product structure on its space of sections. 


\section{Semi-linear representations of Lie groups and action groupoids}

In this section we provide a global formulation in terms of Lie groupoids for the construction described in Section 2: the relationship between derivative representations of Lie algebras on vector bundles and action Lie algebroids has a global analogue, the relationship between the semi-linear representations of Lie groups acting on vector bundles and the action groupoids, described in Theorem 4.8 below. In the following section we shall discuss the more general case of a global formulation for Lie algebroid representations. These global constructions are such that applying the Lie functor to the Lie groups and Lie groupoids under consideration yields the known infinitesimal constructions for Lie algebras and Lie algebroids.

For the basic definitions of groupoid theory used in what follows, see [30] or 28] or [6] (note that the composition convention in [6] is the opposite to that followed here). Given a vector bundle $(E, q, M)$, we denote by $\Phi(E)$ the set of all linear isomorphisms from a fiber of $E$ to some (in general different) fiber of $E$. Then $\Phi(E)$ is a Lie groupoid with base $M$, with its groupoid structure arising from the ordinary composition of maps, and its smooth structure given by locally identifying $\Phi(M \times V)$ with $M \times G L(V) \times M$. (In [30], this groupoid is called the linear frame groupoid of $E$.) The theorem which follows is fundamental to the results of this section.

THEOREM 4.1. The Lie algebroid of the Lie groupoid $\Phi(E)$ is canonically isomorphic to $\mathfrak{D}(E)$.

Theorem 4.1 emerged from two separate lines of work. On the one hand, a proof was given by Kumpera 26 in unpublished notes of 1971, but was not included in the published version (Appendix A of [27]). A simplification of Kumpera's proof was given by Mackenzie in 1987 30, III.4.5]. On the other hand, it was remarked by Hermann in [14 and independently by Kosmann-Schwarzbach in 22 that the derivative endomorphisms of the space of sections of a vector bundle are the infinitesimal generators of one-parameter groups of vector bundle automorphisms. This leads precisely to the result that $A \Phi(E) \cong \mathfrak{D}(E)$.

We now define the semi-linear isomorphisms of $\Gamma E$ and correspondingly the semi-linear representations of a Lie group on the vector bundle, $E$.

Definition 4.2. Let $(E, q, M)$ be a vector bundle. A semi-linear isomorphism of $\Gamma E$ is an $\mathbb{R}$-linear automorphism $\mu$ of $\Gamma E$ such that there exists an $\mathbb{R}$-linear isomorphism, $\mu^{M}$, of $C^{\infty}(M)$ satisfying

$$
\mu(f \psi)=\mu^{M}(f) \mu(\psi),
$$

for all $f \in C^{\infty}(M)$ and all $\psi \in \Gamma E$.

It follows that $\mu^{M}$ is a ring automorphism of $C^{\infty}(M)$. Any automorphism $\nu$ of the vector bundle $E$ that projects onto a transformation $\nu_{M}$ of the base manifold gives rise to a semi-linear isomorphism $\psi \mapsto \nu \cdot \psi$ of $\Gamma E$ defined by

$$
\nu \cdot \psi=\nu \circ \psi \circ\left(\nu_{M}\right)^{-1},
$$

in which case the ring endomorphism of $C^{\infty}(M)$ is $f \mapsto f \circ\left(\nu_{M}\right)^{-1}$, and conversely, every semi-linear isomorphism of $\Gamma E$ arises from a vector bundle automorphism of E. (See 21] 22].) 
Proposition 4.3. Formula (20) establishes a one-to-one correspondence between vector bundle automorphisms of $E$ and semi-linear isomorphisms of $\Gamma E$.

The semi-linear isomorphisms of $\Gamma E$ are the $\mathbb{R}$-isomorphisms of $\Gamma E$ which can be extended to automorphisms of the algebra of sections of the tensor algebra of $E$.

DeFinition 4.4. A semi-linear representation of a Lie group $G$ on $E$ is a morphism, $R$, from $G$ into the group of semi-linear isomorphisms of $\Gamma E$ such that, for any $\psi \in \Gamma E$, the map $(g, m) \mapsto R(g)(\psi)(m), G \times M \rightarrow E$, is smooth.

Each semi-linear representation, $R$, of $G$ on $E$ is associated to an action $g \mapsto g_{M}$ of $G$ on $M$ such that $(R(g))^{M}(f)=f \circ\left(g_{M}\right)^{-1}$.

There is also a well-known notion of a Lie groupoid representation, which we recall.

Definition 4.5. A representation of a Lie groupoid $G$ with base $M$ on the vector bundle $E$ is a base-preserving Lie groupoid morphism from $G$ to $\Phi(E)$.

If the base $M$ is a point, $G$ is merely a Lie group and $E$ is a vector space, and this definition reduces to that of a linear representation. In general it follows from Theorem 4.1 that applying the Lie functor to a representation of $G$ on $E$ yields a representation of $A G$ on $E$.

To express the relationship between the notion of semi-linear representation and that of a groupoid representation, we recall the definition of action groupoid. Consider an action $g \mapsto g_{M}$ of a Lie group $G$ on a manifold $M$. The product manifold $G \times M$ becomes a Lie groupoid with base $M$ when it is equipped with the source map $(g, m) \mapsto m$, the target map $(g, m) \mapsto g_{M}(m)$, for $g \in G, m \in M$, the partial multiplication $\left(\left(g_{1}, m_{1}\right),\left(g_{2}, m_{2}\right)\right) \mapsto\left(g_{1} g_{2}, m_{2}\right)$ defined if and only if $m_{1}=\left(g_{2}\right)_{M}\left(m_{2}\right)$, and the inversion $(g, m) \mapsto\left(g^{-1}, g_{M}(m)\right)$. With this Lie groupoid structure, the product manifold $G \times M$ is called the action groupoid corresponding to the action $g \mapsto g_{M}$, and we denote it by $G \varangle M$. The following result is proved in [17. Th. 2.5].

Proposition 4.6. If $\mathfrak{g}$ is the Lie algebra of the Lie group $G$, the Lie algebroid of the action groupoid $G \varangle M$ is the action Lie algebroid $\mathfrak{g} \varangle M$ associated to the infinitesimal of the action of $G$.

EXAMPLE 4.7. The representation $\sigma$ of the Lie algebroid $\mathfrak{g} \varangle M$ on $E$ of Example 2.5 can be integrated to a morphism $G \varangle M \rightarrow \Phi_{0}(E)$, where $\Phi_{0}(E)$ is the Lie subgroupoid of $\Phi(E)$ of isometries between the fibers of $E$, i.e., to an orthogonal representation of $G \varangle M$ on $E$, if and only if $(E, \nabla)$ together with the metric constitute $G$-invariant data in the sense of 13 .

We can now relate the two concepts defined in 4.4 and 4.5 .

THEOREM 4.8. Let $g \mapsto g_{M}$ be an action of a Lie group $G$ on $M$. There is a oneto-one correspondence between semi-linear representations of $G$ on $E$, associated to the given action, and representations of the action groupoid $G \varangle M$ on $E$.

Proof. The proof follows from the observations that semi-linear representations are in one-to-one correspondence with the actions of $G$ on $E$ by vector bundle automorphisms, which in turn are in one-to-one correspondence with morphisms of Lie groupoids from the action groupoid, $G \varangle M$, to $\Phi(E)$, i.e., representations of the action groupoid on $E[30$. 
In view of the properties of the Lie functor stated in Theorem 4.1 and Proposition 4.6, this theorem implies Theorem 2.4 when the action of $\mathfrak{g}$ on $M$ can be integrated globally.

REMARK 4.9. The semi-linear transformations of $\Gamma E$ are another particular case of the pseudo-linear transformations of Jacobson [20], in which the linear mapping $D_{\mathcal{C}}$ vanishes, and the ring automorphism $f \mapsto \bar{f}$ of $\mathcal{C}=C^{\infty}(M)$ is, in the notation of Definition 4.2, the map $\mu^{M}$.

REMARK 4.10. The isomorphism of Theorem 4.1 arises as an infinitesimal version of the correspondence between vector bundle automorphisms and semi-linear isomorphisms (see Proposition 4.3), but also admits another formulation.

A vector field $X$ on a vector bundle $(E, q, M)$ is linear if the flow of $X$ consists of local vector bundle automorphisms of $E$. Such a vector field clearly projects to a vector field $X_{M}$ on $M$. In [32], linear vector fields are characterized as pairs $X \in \Gamma T E, X_{M} \in \Gamma T M$ which constitute a vector bundle morphism from $(E, q, M)$ to $(T E, T(q), T M)$.

The one-to-one correspondence between linear vector fields on $E$ and derivative endomorphisms of $\Gamma E$ can be expressed directly as follows [23] [32]. Given a vector field $X$ on $E$ which projects to a vector field $X_{M}$ on $M$, and a section $\psi$ of $E$, we define

$$
D_{X}(\psi)(m)=T_{m}(\psi)\left(X_{M}(m)\right)-X(\psi(m)),
$$

for $m \in M$. (Here the right-hand side is a vertical tangent vector, which we regard as an element of $E$ in the usual way.) When $X$ is linear, the $\mathbb{R}$-linear map $D_{X}$ is a derivative endomorphism of $\Gamma E$ associated with $X_{M}$, and is called the Lie derivation with respect to $X$. Conversely, given a derivative endomorphism $D$ of $\Gamma E$ associated with a derivation $X_{M}$ of $C^{\infty}(M)$, we set

$$
X_{D}(p)=T_{m}(\psi)\left(X_{M}(m)\right)-(D \psi)(m),
$$

where $p \in E$ and $\psi$ is a section of $E$ such that $\psi(m)=p$. Then $X_{D}$ is a well-defined linear vector field on $E$, and the maps $X \mapsto D_{X}$ and $D \mapsto X_{D}$ are obviously inverses of one another. Moreover this one-to-one correspondence preserves brackets. In fact, the linear vector fields are the section of a Lie algebroid $T^{\mathrm{LIN}} E$ with base $M$, defined in [32], and this correspondence arises from a Lie algebroid isomorphism from $T^{\mathrm{LIN}} E$ to $\mathfrak{D}(E)$. Therefore the isomorphism of Theorem 4.1 may also be formulated as an isomorphism between the Lie algebroid of $\Phi(E)$ and $T^{\mathrm{LIN}} E$.

An infinitesimal action of a Lie algebroid $A$ on a vector bundle $E$ in the sense of Definition 3.1 is called linear if the action is by linear vector fields. Since a Lie algebroid morphism from $A$ to $T^{\mathrm{LIN}} E$ can be identified with a Lie algebroid morphism from $A$ to $\mathfrak{D}(E)$, it follows that the linear infinitesimal actions of $A$ on $E$ can be identified with the representations of $A$ on $E$ in the sense of Definition 2.3. Since, by condition (i) of Definition 3.1, for each $X \in \Gamma A, X_{M}=a(X)$, the correspondence established by (21) coincides with that of [17, p. 212].

A more general non-linear version of the bijective correspondence between vector fields on $E$ and differential operators on the sections of $E$ was established in 24. Consider a fiber bundle $(F, q, M)$. Then, given an infinitesimal automorphism of the bundle, that is, a vector field $X$ on $F$ which projects to a vector field $X_{M}$ on $M$, and a section $\psi$ of $F$, formula (21) defines a vertical tangent vector to $F$, and we thus obtain a section of $\psi^{*} V F$, where $V F$ is the vertical tangent bundle 
of $F$. In this way one obtains an operator which is a first-order differential section operator from $F$ to $V F$ in the terminology of [34. Conversely, such an operator corresponds to an infinitesimal automorphism if and only if it is quasi-scalar. For a fibered manifold $(F, q, M)$, the set of infinitesimal automorphisms has both a module structure over $C^{\infty}(M)$ and a bracket, making it a Lie pseudo-algebra, but in general there is no underlying Lie algebroid.

\section{Groupoid actions and representations}

More generally, we now consider the action of a Lie groupoid on a fibered manifold. (We shall not consider the still more general case of the action of a Lie groupoid on an arbitrary smooth map. For this, see both [17] and the recent account of Moerdijk and Mrčun 33.)

Let $G$ be a Lie groupoid with base $M$ and source map $\alpha$, and let $\varphi: F \rightarrow M$ be a fibered manifold. We denote by $\varphi^{*} G$ the pullback in the following commutative diagram,

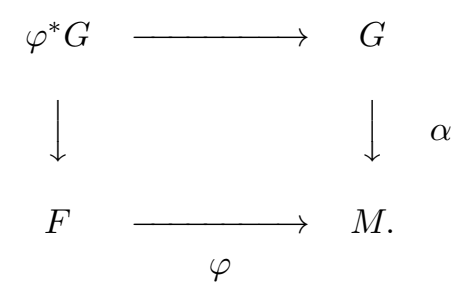

For $m \in M$, we denote $\varphi^{-1}(m) \subseteq F$ by $F_{m}$.

Definition 5.1. Let $G$ be a Lie groupoid with base $M$, source map $\alpha$ and target map $\beta$, and let $\varphi: F \rightarrow M$ be a fibered manifold. An action of $G$ on $F$ is a map, $S$, from $G$ to the set of smooth bijective maps from a fiber of $F$ to some (in general different) fiber of $F$, such that

(i) for any $g \in G, S(g)$ is a map from $F_{\alpha(g)}$ to $F_{\beta(g)}$,

(ii) for any $g_{1} \in G$ and $g_{2} \in G$ such that $\beta\left(g_{1}\right)=\alpha\left(g_{2}\right), S\left(g_{2} g_{1}\right)=S\left(g_{2}\right) \circ S\left(g_{1}\right)$,

(iii) the map $(g, p) \mapsto S(g)(p)$ from $\varphi^{*} G$ to $F$ is smooth.

If, in particular, the base $M$ is a point, then $G$ is a Lie group, and we recover the notion of action of a Lie group on a manifold $F$.

When $F=M$ and $\varphi$ is the identity, the only action of the Lie groupoid $G$ on $M$ is that given by $S(g)(\alpha g)=\beta g$.

It was originally shown by Ehresmann [8] that whenever an action, $S$, of $G$ on $\varphi: F \rightarrow M$ is defined, there is an associated Lie groupoid structure on the manifold $\varphi^{*} G$, with base $F$. The source map is $(g, p) \mapsto p$, while the target map is $(g, p) \mapsto S(g)(p)$. The partial multiplication is $\left(\left(g_{1}, p_{1}\right),\left(g_{2}, p_{2}\right)\right) \mapsto\left(g_{1} g_{2}, p_{2}\right)$ defined if and only if $g_{1}=S\left(g_{2}\right) p_{2}$, and the inversion is $(g, p) \mapsto\left(g^{-1}, S(g)(p)\right)$. With this Lie groupoid structure, $\varphi^{*} G$ is called the action groupoid associated to the given action, and we denote it by $G \varangle F$. The top arrow in the preceding diagram is a morphism of Lie groupoids from $G \varangle F$ to $G$, over $\varphi: F \rightarrow M$.

If $M$ is a point, then the Lie groupoid $G$ is a Lie group, and the Lie groupoid structure defined on $G \times F$ reduces to the action groupoid structure described in Section 1 associated to a Lie group action on a manifold. 
The Lie functor maps action groupoids to action Lie algebroids. More precisely, the differential, $\sigma$, of $S$ is defined by

$$
\sigma(X)(p)=T S(p)\left(X_{\varphi(p)}\right)
$$

where $p \in F$ and $X \in \Gamma A G$, while $T S(p)$ denotes the tangent map at the unity, $\varphi(p) \in M$, of the map $g \mapsto S(g)(p)$ from $G$ to $F$. The result which follows was proved in [17].

Proposition 5.2. Let $S$ be an action of the Lie groupoid $G$ on $\varphi: F \rightarrow M$. Then $S$ differentiates to an infinitesimal action of the Lie algebroid $A G$ on $F$, and the Lie algebroid of the action groupoid $G \varangle F$ is the action Lie algebroid $A G \varangle F$.

Before considering a notion of semi-linear representation for Lie groupoids, we recall the notion of bisection.

DeFinition 5.3. Let $G$ be a Lie groupoid, with base $M$, source map $\alpha$ and target map $\beta$. A map $b: M \rightarrow G$ is a bisection of $G$ if $\alpha \circ b=\operatorname{Id}_{M}$ and $\beta \circ b$ is a diffeomorphism of $M$.

The set of bisections of $G$ is a group under the composition law defined by

$$
\left(b_{1} \cdot b_{2}\right)(m)=b_{1}\left(\left(\beta \circ b_{2}\right)(m)\right) b_{2}(m),
$$

for bisections $b_{1}$ and $b_{2}$, and $m \in M$. The group of bisections of $G$ is denoted by $\mathcal{B}(G)$.

Proposition 5.4. Let $(E, q, N)$ be a vector bundle. There is a canonical group isomorphism from $\mathcal{B}(\Phi(E)$ ) to the group of vector bundle automorphisms of $E$.

Proof. Given a bisection, $b$, of $\Phi(E)$, we define a bundle map, $\tilde{b}$, from $E$ to itself by

$$
\tilde{b}(v)=b(q(v))(v),
$$

for $v \in E$. Then $\tilde{b}$ is a vector bundle automorphism of $E$, projecting onto the diffeomorphism $\beta \circ b$ of $N$. Conversely, given a vector bundle automorphism $u$ of $E$, the map $\left.x \mapsto u\right|_{E_{x}}, x \in N$, defines a bisection of $\Phi(E)$. The smoothness of the map is proved by using the local triviality of $E$. These maps are mutually inverse and they are group morphisms.

It follows from Proposition 4.3 that $\mathcal{B}(\Phi(E))$ is isomorphic to the group of semi-linear isomorphisms of $\Gamma E$.

Now consider Lie groupoids $G_{1}$ and $G_{2}$ on the same base $M$. It is clear that any Lie groupoid morphism $u: G_{1} \rightarrow G_{2}$ over $M$ defines a group morphism $U: \mathcal{B}\left(G_{1}\right) \rightarrow \mathcal{B}\left(G_{2}\right)$ of the corresponding groups of bisections by

$$
U(b)(m)=u(b(m)),
$$

for $b \in \mathcal{B}\left(G_{1}\right)$ and $m \in M$. Thus a semi-linear representation of a Lie group $G$ on a vector bundle $(E, q, M)$ may be regarded as a group morphism from $\mathcal{B}(G)$ to $\mathcal{B}(\Phi(E))$ which satisfies the smoothness condition in 4.4.

Let $G$ be a Lie groupoid acting on a fibered manifold, $\varphi: F \rightarrow M$, with action groupoid $G \varangle F$. We consider a vector bundle $E$ on base $F$ and a representation of $G \varangle F$ on $E$, i.e., a morphism of Lie groupoids from $G \varangle F$ to $\Phi(E)$. For groupoids in the category of sets, this notion was studied by Brown [5]. In the smooth case, such a representation is a global form of a derivative representation. Indeed, by 
Proposition 5.2 it differentiates to a representation of $A G \varangle F$ on $E$, which by Theorem 3.5 is a derivative representation of $A G$ on $E$.

Thus for each $g \in G$ and $p \in F_{\alpha g}$, we are given an isomorphism from $E_{p}$ to $E_{S(g) p}$, where $S$ is the given action of $G$ on $F$. According to (23) and to Proposition 5.4, there is a group morphism from $\mathcal{B}(G \varangle F)$ to the group of semi-linear isomorphisms of $\Gamma E$. In addition, given a bisection $b$ of $G$, there is a bisection $\hat{b}$ of $G \varangle F$ defined by

$$
\hat{b}(p)=(b(\varphi(p)), p)
$$

for $p \in F$, and the map $b \mapsto \hat{b}$ is a group morphism from $\mathcal{B}(G)$ to $\mathcal{B}(G \varangle F)$. Composing these maps we obtain a group morphism from $\mathcal{B}(G)$ to the group of semi-linear isomorphisms of $\Gamma E$. This group morphism, $R$, is associated with the given action of $G$ on $F$, in the sense that if $v \in E_{p}$ for $p \in F$, then $R(b) v \in E_{p^{\prime}}$, where $p^{\prime}=S(b(\varphi p)) p$, for all $b \in \mathcal{B}(G)$. Thus we have:

Proposition 5.5. Given an action of a Lie groupoid $G$ on a fibered manifold $\varphi: F \rightarrow M$, to any representation of $G \varangle F$ on a vector bundle $E$ with base $F$ there corresponds a representation of the group of bisections of $G$ by semi-linear isomorphisms of $\Gamma E$, associated to the given action.

Since the Lie algebra of sections of $A G$ can be formally regarded as the Lie algebra of the infinite-dimensional group $\mathcal{B}(G)$, this proposition can be viewed as a global form of part of Proposition 3.5. When the Lie groupoid $G$ is a Lie group, its group of bisections is the group itself, so this proposition is a partial generalization of Theorem 4.8 to the case of Lie groupoids. We may therefore regard the group morphisms from $\mathcal{B}(G)$ to the group of semi-linear isomorphisms of $\Gamma E$ satisfying a suitable smoothness condition as the semi-linear representations of $G$ on $E$. However, the characterization of smoothness for such semi-linear representations of groupoids in terms of the bisections of $G$ alone appears to be a difficult matter. The concept of bisection may readily be localized, leading to a sheaf of germs of local bisections, but the relationship between bisections of $G$ and bisections of $G \varangle F$ evades a simple description.

Remark 5.6. The representation of the group of bisections in Proposition 5.5 may be approached in an alternative way. We consider a representation of $G \varangle F$ on $E$ as above and, for $g \in G$ and $p \in F_{\alpha g}$, we denote the isomorphism from $E_{p}$ to $E_{S(g) p}$ by $\rho(g, p)$. Given $g \in G$, to any section $\psi$ of $E$ over $F_{\alpha g}$ we can associate a section $g \cdot \psi$ of $E$ over $F_{\beta g}$, by

$$
(g \cdot \psi)(p)=\rho\left(g, S\left(g^{-1}\right) p\right)\left(\psi\left(S\left(g^{-1}\right) p\right)\right),
$$

for $p \in F_{\beta g}$. The map $\psi \mapsto g \cdot \psi$ is semi-linear with respect to the action $S$,

$$
(g \cdot(f \psi))(p)=f\left(S\left(g^{-1}\right) p\right)(g \cdot \psi)(p),
$$

for $f \in C^{\infty}\left(F_{\alpha g}\right)$. By allowing $g$ to range through the values of a bisection, we obtain a semi-linear isomorphism of $\Gamma E$ : take any $b \in \mathcal{B}(G)$ and for $\psi \in \Gamma E$ and $p \in E$ define

$$
(b \cdot \psi)(p)=(g \cdot \psi)(p)
$$

where $g=b\left((\beta \circ b)^{-1}(\varphi(p))\right)$. It is clear that the resulting map from $\mathcal{B}(G)$ to the group of semi-linear isomorphisms of $\Gamma E$ is the same as that of Proposition 5.5. This formulation perhaps shows more clearly the effect of the action on sections of 
$E$, but it is still unclear how to characterize the smoothness of $\rho$ in terms of the maps $\psi \mapsto g \cdot \psi$.

\section{Appendix: some algebraic formulations}

As we remarked in the introduction, both the derivative endomorphisms and the semi-linear isomorphisms are particular cases of the pseudo-linear transformations in the sense of Jacobson. Here, we characterize the pseudo-linear endomorphisms of a left module $\mathcal{E}$ over a ring $\mathcal{C}$ in terms of twisted derivations. In particular, the derivative endomorphisms are characterized in terms of derivations. We also characterize the semi-linear isomorphisms in terms of algebra automorphisms. The case that is relevant to differential geometry is that of $\mathcal{E}=\Gamma E$ and $\mathcal{C}=C^{\infty}(M)$, where $E$ is a vector bundle over a manifold $M$. For simplicity, we shall formulate the results in this case only.

The notion of derivation of an algebra can be generalized as follows.

Definition 6.1. Let $\mathcal{A}$ be an algebra and let $\alpha$ be a linear endomorphism of $\mathcal{A}$. A twisted derivation of $\mathcal{A}$ with respect to $\alpha$ is a linear endomorphism $\mathcal{U}$ of $\mathcal{A}$ such that, for all $a, b \in \mathcal{A}$,

$$
\mathcal{U}(a b)=\mathcal{U}(a) b+\alpha(a) \mathcal{U}(b) .
$$

It follows from (25) that, if $\operatorname{Im}(\mathcal{U})$ has an element which is not a torsion element, the map $\alpha$ is an algebra endomorphism.

Definition 6.2. An $\mathbb{R}$-linear endomorphism $u$ of $\Gamma E$ is called pseudo-linear if there exist $\mathbb{R}$-linear endomorphisms $u^{M}$ and $u_{M}$ of $C^{\infty}(M)$ such that

$$
u(f \psi)=u^{M}(f) u(\psi)+u_{M}(f) \psi,
$$

for all $f \in C^{\infty}(M)$ and $\psi \in \Gamma E$.

It follows from (26) that, whenever there exists $\psi \in \Gamma E$ such that $u(\psi)$ and $\psi$ are linearly independent over the ring $C^{\infty}(M)$, (i) $u^{M}$ is an algebra endomorphism of $C^{\infty}(M)$, and (ii) $u_{M}$ is a twisted derivation of $C^{\infty}(M)$ with respect to $u^{M}$.

Clearly the derivative endomorphisms (resp., the semi-linear isomorphisms) of $\Gamma E$ are the pseudo-linear endomorphisms for which $u^{M}$ is the identity of $C^{\infty}(M)$ (resp., which are bijective and for which $u_{M}=0$ ).

Following Grothendieck [12, 16.5], we let $\mathcal{A}(E)$ be the vector space $C^{\infty}(M) \oplus$ $\Gamma E$ with the commutative $\mathbb{R}$-algebra structure such that the product of any two elements of $C^{\infty}(M)$ is their product in the ring $C^{\infty}(M)$, the product of $f \in C^{\infty}(M)$ and $\psi \in \Gamma E$ is $f \psi$ in the $C^{\infty}(M)$-module $\Gamma E$, and the product of any two sections of $\Gamma E$ vanishes. We can now formulate the following results, whose proofs are all straightforward computations.

By a slight abuse of language, we let $u^{M}$ also denote the endomorphism of $\mathcal{A}(E)$ which coincides with $u^{M}$ on $C^{\infty}(M)$ and vanishes on $\Gamma E$.

Proposition 6.3. Let $u^{M}: C^{\infty}(M) \rightarrow C^{\infty}(M), u_{M}: C^{\infty}(M) \rightarrow C^{\infty}(M)$ and $u: \Gamma E \rightarrow \Gamma E$ be $\mathbb{R}$-linear maps. Then $u_{M}+u$ is a twisted derivation of the $\mathbb{R}$-algebra $\mathcal{A}(E)$ with respect to $u^{M}$ if and only if (i) $u_{M}$ is a twisted derivation of $C^{\infty}(M)$ with respect to $u^{M}$, and (ii) $u$ is a pseudo-linear endomorphism of $\Gamma E$ with associated endomorphisms of $C^{\infty}(M), u^{M}$ and $u_{M}$.

When $u^{M}$ is the identity of $C^{\infty}(M)$, the twisted derivations are ordinary derivations, so we obtain the following corollary. 
Proposition 6.4. Let $u_{M}: C^{\infty}(M) \rightarrow C^{\infty}(M)$ and $u: \Gamma E \rightarrow \Gamma E$ be $\mathbb{R}$-linear maps. Then $u_{M}+u$ is a derivation of the $\mathbb{R}$-algebra $\mathcal{A}(E)$ if and only if (i) $u_{M}$ is a derivation of $C^{\infty}(M)$, and (ii) $u$ is a derivative endomorphism of $\Gamma E$ with associated derivation of $C^{\infty}(M), u_{M}$.

An analogous characterization holds for the semi-linear isomorphisms, in terms of algebra automorphisms.

Proposition 6.5. Let $u^{M}: C^{\infty}(M) \rightarrow C^{\infty}(M)$ and $u: \Gamma E \rightarrow \Gamma E$ be $\mathbb{R}$-linear isomorphisms. Then $u^{M}+u$ is an algebra automorphism of $\mathcal{A}(E)$ if and only if (i) $u^{M}$ is an algebra automorphism of $C^{\infty}(M)$, and (ii) $u$ is a semi-linear isomorphism of $\Gamma E$ with associated automorphism of $C^{\infty}(M), u^{M}$.

The vector space of pseudo-linear endomorphisms of $\Gamma E$ is not closed under composition, but it follows from Proposition 6.5 that the semi-linear isomorphisms of $\Gamma E$ form a group. In addition, just like the set of twisted derivations, the set of pseudo-linear endomorphisms is not closed under commutators, but it follows from Proposition 6.4 that the derivative endomorphisms of $\Gamma E$ form a Lie algebra.

\section{References}

[1] M. F. Atiyah. Complex analytic connections in fibre bundles. Trans. Amer. Math. Soc., 85:181-207, 1957.

[2] C. M. de Barros. Espaces infinitésimaux. Cahiers Topologie Géom. Différentielle, 7, 1964. xi +96 pages.

[3] A. A. Beilinson and V. V. Schechtmann. Determinant bundles and Virasoro algebras. Comm. Math. Phys., 118:651-701, 1988.

[4] N. Bourbaki. Algèbre. Chapîtres 1-3. Hermann, Paris, 1970.

[5] R. Brown. Fibrations of groupoids. J. Algebra, 15:103-132, 1970.

[6] A. Cannas da Silva and A. Weinstein. Geometric models for noncommutative algebras, volume 10 of Berkeley Mathematics Lecture Notes. American Mathematical Society, 1999.

[7] P. Dazord. Groupoïde d'holonomie et géométrie globale. C. R. Acad. Sci. Paris, Série A, 324:77-80, 1997.

[8] C. Ehresmann. Gattungen von lokalen strukturen. Jahresber. d. Deutschen Math. Verein., 60:49-77, 1957. Contained in 9], Partie II-1. French translation, Espèces de structures locales. Séminaire Topologie Géom. Différentielle, 3, 1962. 24 pages.

[9] C. Ehresmann. Euvres complètes et commentées, A. C. Ehresmann, editor. Seven volumes. Imprimerie Evrard, Amiens, 1984

[10] S. Evens, Jiang-Hua Lu, and A. Weinstein. Transverse measures, the modular class and a cohomology pairing for Lie algebroids. Quart. J. Math. Oxford Ser. (2), 50(200):417-436, 1999.

[11] G. L. Fel'dman. Global dimension of rings of differential operators. Trans. Moscow Math. Soc., 41(1):123-147, 1982.

[12] A. Grothendieck. Éléments de géométrie algébrique. IV. Étude locale des schémas et des morphismes de schémas. Publ. Math. Inst. Hautes Études Sci., no. 32, 1967.

[13] V. Guillemin and S. Sternberg. Geometric quantization and multiplicities of group representations. Invent. Math., 67:515-538, 1982.

[14] R. Hermann. Vector Bundles in Mathematical Physics, Vol. 1. Benjamin, New York, 1970.

[15] J.-C. Herz. Pseudo-algèbres de Lie. C. R. Acad. Sci. Paris, Série A, 236:1935-1937, 1953.

[16] J.-C. Herz. Pseudo-algèbres de Lie, II. C. R. Acad. Sci. Paris, Série A, 236:2289-2291, 1953.

[17] P. J. Higgins and K. C. H. Mackenzie. Algebraic constructions in the category of Lie algebroids. J. Algebra, 129:194-230, 1990.

[18] J. Huebschmann. Poisson cohomology and quantization. J. Reine Angew. Math., 408:57-113, 1990.

[19] N. Jacobson. On pseudo-linear transformations. Proc. Nat. Acad. Sci., 21:667-670, 1935.

[20] N. Jacobson. Pseudo-linear transformations. Ann. Math., 38:485-506, 1937. 
[21] Y. Kosmann. Groupes de transformations et covariance des opérateurs différentiels. C. $R$. Acad. Sci. Paris, Série A, 275:1235-1237, 1972.

[22] Y. Kosmann. On Lie transformation groups and the covariance of differential operators. In Differential geometry and relativity, pages 75-89. Mathematical Phys. and Appl. Math., Vol. 3. Reidel, Dordrecht, 1976.

[23] Y. Kosmann-Schwarzbach. Dérivées de Lie des morphismes de fibrés. In Differential geometry (Paris, 1976/1977), pages 55-71. Univ. Paris VII, Paris, 1978.

[24] Y. Kosmann-Schwarzbach. Vector fields and generalized vector fields on fibered manifolds. In Geometry and differential geometry (Proc. Conf., Univ. Haifa, 1979), pages 307-355. Lect. Notes Math., Vol. 792, Springer, Berlin, 1980.

[25] J.-L. Koszul. Lectures on fibre bundles and differential geometry. Notes by S. Ramanan. Tata Institute of Fundamental Research, Bombay, 1960.

[26] A. Kumpera. An introduction to Lie groupoids. Duplicated notes, Núcleo de Estudos e Pesquisas Científicas, Rio de Janeiro, 1971.

[27] A. Kumpera and D. C. Spencer. Lie equations. Volume I: General theory. Princeton University Press, 1972.

[28] N. P. Landsman. Mathematical topics between classical and quantum mechanics. SpringerVerlag, New York, 1998.

[29] K. Mackenzie. Cohomology of locally trivial groupoids and Lie algebroids. PhD dissertation, Monash University, Department of Mathematics, 1979. xi + 203pp.

[30] K. Mackenzie. Lie groupoids and Lie algebroids in differential geometry. London Mathematical Society Lecture Note Series, no. 124. Cambridge University Press, Cambridge, 1987.

[31] K. C. H. Mackenzie. Lie algebroids and Lie pseudoalgebras. Bull. London Math. Soc., 27(2):97-147, 1995.

[32] K. C. H. Mackenzie and Ping Xu. Classical lifting processes and multiplicative vector fields. Quarterly J. Math. Oxford (2), 49:59-85, 1998.

[33] I. Moerdijk and J. Mrčun. On integrability of infinitesimal actions. Amer. J. Math., 124(3):567-593, 2002.

[34] R. S. Palais. Foundations of global non-linear analysis. W. A. Benjamin, Inc., New YorkAmsterdam, 1968.

[35] J. Pradines. Théorie de Lie pour les groupoïdes différentiables. Calcul différentiel dans la catégorie des groupoïdes infinitésimaux. C. R. Acad. Sci. Paris, Série A, 264:245-248, 1967.

[36] Ngô Van Quê. Du prolongement des espaces fibrés et des structures infinitésimales. Ann. Inst. Fourier (Grenoble), 17:157-223, 1967.

[37] Ngô Van Quê. Sur l'espace de prolongement différentiable. J. Differential Geom., 2:33-40, 1968.

[38] Ngô Van Quê. Nonabelian Spencer cohomology and deformation theory. J. Differential Geom., 3:165-211, 1969.

[39] N. Reshetikhin, A. Voronov, and A. Weinstein. Semiquantum geometry. J. Math. Sci., 82(1):3255-3267, 1996.

[40] Ping Xu. Gerstenhaber algebras and BV-algebras in Poisson geometry. Comm. Math. Phys., 200(3):545-560, 1999.

Centre de Mathématiques, UMR 7640 du CNRS, École Polytechnique, F-91128, Palaiseau, France

E-mail address: yks@math.polytechnique.fr

Department of Pure Mathematics, University of Sheffield, Sheffield, S3 7RH, UNITED KINGDOM

E-mail address: K.Mackenzie@sheffield.ac.uk 